\title{
Per una presa di posizione che non riduca il dibattito alcologico ai soli temi epidemiologici
}

\author{
Enrico Tempesta, Michele Contel
}

Da più di 25 anni I'Osservatorio Permanente sui Giovani e I'Alcol (OPGA) lavora con i ricercatori e gli operatori sul tema della conoscenza e dell'intervento relativi al consumo e all'abuso di bevande alcoliche nelle fasce giovanili. In questi anni abbiamo proposto all'attenzione del pubblico una lettura complessa e interdisciplinare del fenomeno cercando sempre di registrare le dinamiche sociali soggiacenti a comportamenti di addiction.

Nel documento che segue il Laboratorio Scientifico dell'Associazione ha elaborato un position paper su tre temi che è stato discusso e redatto interdisciplinarmente e diffuso ad una platea di esperti scientifici e operatori allo scopo di dialogare con un pubblico di lettori indipendenti e, al tempo stesso, profondamente coinvolti nella vicenda della ricerca e della cura nei settori delle dipendenze e specificamente di quelle connesse all'abuso di alcolici.

Il documento affronta tre temi: 1) la proposta di un eventuale innalzamento dell'età minima legale per l'acquisto e il consumo di bevande alcoliche dai 18 ai 25 anni; 2) la dose soglia compatibile con un consumo non a rischio o a rischio molto basso; 3 ) la grandezza accettabile del rischio del bere rispetto ad altri rischi.

L'esercizio che i membri del Laboratorio Scientifico si sono attribuiti consiste in una riflessione sulla praticabilità dei concetti dell'alcologia sociale in ambiti solitamente presidiati dall'epidemiologia medica. Ragionare in una prospettiva ampia è doveroso per il nostro lavoro. Il confronto sui tre temi che presentiamo pone problemi metodologici e di contenuto di primaria importanza e di non minore complessità. Abbiamo scelto tre temi "scomodi" per un approccio di tipo sociologico, proprio perché riteniamo che guardare oltre le inevitabili abitudini e inerzie che ogni metodo comporta significa rinnovare la sfida e accettare le provocazioni di altri approcci e modi di pensare.

Discutere sul valore preventivo dell'innalzamento dell'età legale vuole evidenziare le contraddizioni di una prassi comune e diffusa nel mondo occidentale. I giovani cominciano a bere precocemente, sotto i 18 anni (in media, secondo i dati ESPAD 2015 relativi a 26 Paesi, il 47\% a 13 anni ha già consumato un drink). È perciò necessario interrogarsi su quali siano i mezzi più adeguati per mitigare i rischi di un approccio precoce, senza "traghettare" nel campo della dipendenza simpliciter momenti della transizione adolescenziale che non sono predittivi di conse- guenze avverse. II tema del rischio accettabile si interroga sulla questione di se e quanto la nozione di rischio può essere suscettibile di una gestione attiva di tipo soggettivo al di là delle raccomandazioni tipo che sono proprie delle linee guida in ambito sanitario. Infine, la riflessione sul bilanciamento dei diversi rischi all'interno dei comportamenti individuali e collettivi intende porre l'attenzione sul fatto che gli individui e le famiglie sono oggi assediati da messaggi di tipo ortoressico che toccano tutti i comportamenti legati all'alimentazione e in generale alla ricerca di stili di vita più controllati. Tutto ciò mentre in Italia la curva dei consumi e degli abusi di bevande alcoliche è stabilmente in discesa. E tuttavia le norme vanno nella direzione di rendere sempre più presente la preoccupazione della consapevolezza del consumatore anche con ipotesi di regolazione prescrittiva delle scelte individuali.

Queste evidenze, riteniamo, meritano una riflessione allargata in un momento storico che vede una risorgenza di conflitti e tensioni anche tra i ricercatori. Ci pare infatti che il tono del dibattito dominante, anche italiano, rifletta una radicalizzazione delle posizioni. Le giuste battaglie di prevenzione, crediamo, non si limitano ad acquisire per sola via di autorità una posizione "olimpica" che pretende di governare i processi a partire dal risultato atteso. È essenziale promuovere il raccordo con I'opportunità dei processi reali di investimento in salute e di moltiplicazione dei criteri dell'azione preventiva.

In materia di consapevolezza del bere molti passi in avanti possono essere compiuti investendo in modo ragionato su fattori persuasivi addizionali alla sola informazione scientifica sui rischi evitabili.

In questo spirito è con piacere che ringraziamo dell'ospitalità il comitato di direzione di Mission per averci consentito di rendere i lettori della rivista parte di questa iniziativa, già cofirmata da 60 operatori ed esperti ai quali vorremmo si aggiungessero quei lettori che ne vogliano condividere i contenuti.

Enrico Tempesta Michele Contel

Tutti coloro che fossero interessati a sottoscrivere il documento possono farlo accedendo al sito www.alcol.net. Per informazioni: osserva.giovani@alcol.net. 


\section{L'alcologia sociale sulle soglie di rischio e l'età legale al bere}

\section{Introduzione}

0.1 A fronte della recente intensificazione, da parte dei media, della comunicazione sui rischi per la salute derivanti dall'uso di bevande alcoliche, I'OPGA nel suo insieme e i membri del Laboratorio Scientifico ritengono di proporre all'attenzione generale alcune riflessioni critiche.

0.2 Questa intensificazione della comunicazione, focalizzata sull'enfasi dei fattori di rischio, si concretizza in un irrigidimento delle raccomandazioni sanitarie riguardo a questioni come: la soglia di età minima legale per il consumo di bevande alcoliche, la soglia di consumi quotidiani compatibili con un rischio di salute da parte di persone sane, I'uso di bevande alcoliche in età giovanile, le restrizioni all'accesso delle bevande, la restrizione alla comunicazione commerciale e l'adozione di politiche fiscali volte a condizionare le scelte del consumatore.

0.3 Le evidenze scientifiche, soprattutto in materia di fattori di rischio dato per scontato che l'obiettivo di ridurre i rischi associati all'abuso di bevande alcoliche è pienamente condiviso spesso non hanno come riferimento l'uomo nella sua interezza. Si configura dunque una situazione paradossale: in nome del rigore scientifico, non vengono prese in considerazione quelle variabili che - come l'ormai ampia letteratura socio-antropologica ha dimostrato - maggiormente condizionano i nostri comportamenti, come ad esempio variabili psicologiche, economiche e sociali e fattori che interessano la più ampia sfera dell'abuso, da un lato, e del comportamento di consumo alimentare, dall'altro. Riteniamo che formulare una soluzione unica valida per tutti, stante la complessità del problema, mancherebbe il bersaglio.

0.4 Nell'analisi del rapporto tra alcol e salute, porre l'enfasi sulla sostanza può condurre a identificare la promozione della salute con modelli solo prescrittivi di regolazione dei comportamenti, a discapito di azioni di prevenzione realizzate in base alle risorse e alle competenze delle persone e dei gruppi sociali ${ }^{1}$.

\section{L'alcol in Italia: fra dato e significato}

1.1 In Italia, è ampio il consenso sul livello dei consumi di bevande alcoliche ${ }^{2}$. I dati di prevalenza, sia pure di varia fonte, sono indiscutibilmente convergenti 3 : una tendenza consolidata alla riduzione dei consumi a partire dagli anni Settanta. Anche gli abusi sono in riduzione, sebbene tale dato vada modulato con attenzione ai diversi periodi e ai diversi profili della popolazione.

1.2 Il danno alcol-correlato è un problema reale e conosciuto da tempo. Tutte le società prevedono un codice sanitario atto a contrastare e ridurre gli effetti negativi del bere. I progressi della medicina negli ultimi 150 anni hanno prodotto un grande avanzamento nella conoscenza dei danni da alcol e nella cura e/o riduzione dei gravi effetti fisici e psichici derivanti dall'abuso, dalla dipendenza e dall'uso improprio in date circostanze. Tuttavia, non si può trascurare una conoscenza non meno importante: i comportamenti del bere sono espressione di complessi assetti socioculturali maturati nel corso dei secoli. In Italia e nei Paesi del Sud Europa, in modo assai più marcato di quanto si evidenzi in altri Paesi, la cultura ha prodotto uno stile del bere contraddistin- to da competenze auto-regolative di tipo collettivo. Queste si riflettono in abitudini consolidate e diffuse del bere in contesti sociali e familiari, che appaiono avere una parte rilevante nella riduzione dei consumi alcolici e dei danni alcol-correlati ${ }^{4}$.

1.3 Pertanto le politiche di regolazione e controllo derivanti da evidenze scientifiche prodotte prevalentemente nei Paesi scandinavi e anglosassoni non possono essere tout court applicate a tutti gli altri Paesi e in particolare all'Italia e ai Paesi del Sud Europa. Infatti I'Italia è un caso significativamente diverso di equilibrio nella gestione del rapporto tra alcol e salute. Esistono molte ragioni di natura sociale e culturale che contribuiscono a spiegare l'anomalia italiana rispetto al profilo di altri Paesi, in quanto fortemente caratterizzata da consumi e da costi sociali decrescenti nel tempo ${ }^{5}$. È necessario studiare e comprendere questa singolarità e continuare a diffondere con convinzione le ragioni che stanno alla base della spiegazione del fenomeno.

\section{I temi critici del dibattito: I'innalzamento del- l'età legale, la dose soglia di consumo, il rischio accettabile, l'efficacia delle politiche restrittive}

2.1 La prima questione sulla quale si sta concentrando una parte dell'alcologia medica riguarda l'indicazione, proposta da alcuni alcologi, concernente l'innalzamento dell'età legale del bere dai 18 ai 25 anni $^{6}$. Si afferma che esistono fondati motivi nella ricerca scientifica più avanzata che raccomandano il ritardo nel contatto con le bevande alcoliche fino al compimento del 25 anno di età. II fondamento scientifico di questa raccomandazione risiederebbe nella disposizione dell'etanolo ad alterare/modificare la plasticità cerebrale dell'individuo. Tale interferenza andrebbe a toccare in profondità vari meccanismi con il rischio di produrre danni neurologici rilevabili.

2.2 $\mathrm{Al}$ centro è il fenomeno del pruning, un processo di rimodellamento sinaptico cerebrale che agisce nella direzione della formazione di sinapsi inibitorie prefrontali tra i 15-20 anni e di sviluppo delle aree sotto-corticali che contribuiscono a dar forma al comportamento caratteristico dell'adolescenza: cioè il passaggio da un substrato essenzialmente emotivo ad uno più razionale e controllato. L'alcol interverrebbe quindi come fattore di alterazione dell'orientamento dello sviluppo fisiologico cerebrale?

2.3 Viene dunque ipotizzata l'idea di un bere comunque non sicuro sino all'età di 25 anni. Tuttavia, si tratterebbe di quantificare nei vari periodi della vita il peso di tale evoluzione, che è molto rapida sino ai cinque anni e presumibilmente modesta in seguito, tanto da rendere problematica l'apposizione di un cut off. Inoltre, se i meccanismi del cosiddetto rewarding sono comuni a molte altre azioni sia di tipo chimico sia comportamentale, risulterebbe necessario inibire un contatto precoce con molte altre sostanze e comportamenti associati.

2.4 Il limite di età può essere discusso nell'ambito di un discorso globale rivolto ai giovani circa il loro stile di vita. Sappiamo quanto vi possa essere di subdolo e ingannevole nell'uso di alcol da parte dei giovani che, attraverso la disforia e/o l'euforia alcolindotte, possono trasformare tale sostanza in droga di iniziazione, di accompagnamento e/o di passaggio. La ridotta percezio- 
ne degli stimoli negativi, in presenza di una più alta sensibilità all'effetto gratificante potrebbe contribuire alla propensione degli adolescenti ad associare più benefici e meno costi all'uso di alcol e di droghe, come anche ad altri comportamenti a rischio. Proprio in questo senso è interesse pubblico proporre ai giovani un modello di vita che non porti a considerare l'alcol una droga o un surrogato di essa.

2.5 La questione non riguarda l'esistenza o meno di un limite di età. Un limite di età deve essere posto purché sia chiaro che tale limite ha una valenza essenzialmente legale e convenzionale, ma non esaurisce il compito di attribuzione di senso entro il quadro dei significati sociali e culturali che anche le Istituzioni hanno il compito di promuovere. Si dovrebbe perciò soprattutto ridurre la vulnerabilità individuale "di stato" conseguente a traumi, stress, disturbi e così via. Piuttosto che vietare l'alcol sino al venticinquesimo anno, lo Stato dovrebbe porre le condizioni che favoriscano contesti educativi dove sia possibile esercitare meglio le funzioni esecutive, imparare a controllare emozioni, pulsioni e appetiti, sviluppando autonomia e responsabilità, anche tramite appositi training. Proprio perché, in virtù della neuroplasticità, il cervello diventa "quello che gli fai fare" ${ }^{8}$. L'attuale soglia dei 18 anni ha un valore legale e convenzionale sensato, perché definisce nella maggior parte dei Paesi europei I'ingresso nella maggiore età e nelle relative responsabilità e diritti. A 18 anni si può votare e guidare un autoveicolo, si entra nella piena responsabilità giuridica e si possono esercitare la patria potestà e i diritti civili.

2.6 Difendere una versione giuridica e convenzionale del requisito dell'età minima legale significa anche tenere conto di alcune implicazioni socioculturali del tema alcol. L'inizio del bere è essenzialmente un'esperienza regolata sullo sfondo di un contesto culturale condiviso. Le figure familiari, i gruppi dei pari, e gli stili con cui si apprende a bere definiscono in profondità il significato e la memoria delle bevande alcoliche nelle comunità umane. La trasmissione intergenerazionale di questi significati, pur in circostanze ormai distanti rispetto alle società tradizionali, costituisce ancora in Italia una ritualità di cui le giovani generazioni beneficiano in senso protettivo.

2.7 Un secondo tema al centro del dibattito riguarda la definizione, nelle raccomandazioni dei comportamenti di salute, di una dose soglia di consumo di bevande alcoliche compatibile con un rischio basso o comunque accettabile, in base alla quale si individuano le popolazioni a rischio e si standardizzano gli strumenti epidemiologici.

2.8 Nell'ambito delle raccomandazioni contenute in documenti controllati da gran parte della comunità scientifica, si afferma che in base a recenti acquisizioni la soglia entro cui è possibile bere con rischio minimo si attesta in due unità alcoliche al giorno per i maschi ed una per le femmine ${ }^{9}$. È bene ricordare che tale soglia è da ritenersi associata al massimo beneficio secondo la distribuzione a "J", almeno per quanto riguarda il beneficio cardiovascolare e l'effetto sulla mortalità alcol correlata.

2.9 La discussione sulle soglie implica una ricaduta immediata sulla nozione di comportamento a rischio e sulla nozione di consumo a rischio (hazardous alcohol consumption), in quanto questi range sono parametrati e, in concreto, applicati a partire dalla definizione di una soglia.

2.10 Da parte di alcuni si dichiara senza remore l'inesistenza di soglie a rischio zero, ribadendo che non esistono evidenze scien- tifiche sul livello di consumo di alcol da ritenersi non rischioso perché ogni livello di consumo di alcol comporta un rischio.

$2.11 \mathrm{Ci}$ sono almeno tre interrogativi riguardo al rischio, che andrebbero ampiamente discussi: (i) il rischio può essere mitigato ma non del tutto eliminato?

(ii) la nozione di rischio è sempre relativa?

(iii) in rapporto ai comportamenti di cui ci stiamo occupando la nozione di rischio deve tenere conto del cosiddetto "rischio deliberativo", categoria che copre quegli aspetti del rischio che dipendono dalla libertà delle persone e dal bilanciamento individuale dei costi e benefici di una situazione data (come avviene anche per il rischio ambientale, alimentare, automobilistico, ecc.)?

2.12 Orientare le scelte di salute pubblica verso un obiettivo di eliminazione completa del rischio o di totale assenza di malattia, senza considerare la dimensione sociale della salute, significa perciò affrontare solo parzialmente il problema e rinunciare ad una considerazione dell'uomo come animale sociale ma solo "come somma grossolana dei suoi organi".

2.13 Un terzo punto riguarda la grandezza accettabile del rischio del bere rispetto ad altri rischi.

2.14 Si fa strada l'assunto che la potenzialità tossica di una sostanza condizioni in modo drastico il ruolo che essa esercita sull'equilibrio Habitat-Soma-Psiche. Jurgen Rehm sostiene che nel mondo occidentale la società abbia un concezione severa del rischio per esposizioni non cercate (aria, acqua, ambiente) e abbia una concezione molto più indulgente per il rischio derivante da esposizioni "volontarie" quali il bere.

2.15 È noto che ogni persona ha una sua individualità biologica che porta a gestire in modo strettamente personale la predisposizione a metabolizzare l'etanolo: le concentrazioni alcolemiche di ogni individuo a fronte della stessa quantità di alcol non sono mai le stesse. Peraltro, come è noto, l'alcol non segue una relazione lineare tra dose e risposta.

2.16 Gli studi epidemiologici circa uso/abuso di alcol e malattia e/o mortalità globale, riportano una finestra di consumo che può far parte di uno stile di vita sano. Infatti, di fronte ai cosiddetti effetti tossici10 derivanti dall'abuso quali:

- maggiore esposizione all'insorgenza di alcuni tumori (in particolare: esofago, faringe, laringe e mammella);

- danni gastrointestinali ed epatici e a carico di altri apparati quali cuore, polmone e rene;

- labilità emotiva;

- vari impairment neurocognitivi legati alla progressiva atrofia cerebrale;

- e, non meno grave, lo sviluppo della dipendenza alcolica non si possono non menzionare, in un'ottica di bilanciamento, i ben noti effetti cosiddetti "benefici" sulla salute11, derivanti dalI'uso delle bevande alcoliche in quantità moderate fra i quali:

- miglioramento di quelli che sono i fattori di rischio metabolici correlati a CVD (cardiovascular diseases), inclusa la riduzione del rischio di morbilità e mortalità per CHD (coronary heart disease);

- miglioramento del profilo lipidico (incrementa in maniera dose-dipendente i livelli di HDL-c e Apo-Al ma senza alterare il livelli di LDL-c);

- sensibilità all'insulina e riduzione del rischio di diabete mellito di tipo II;

- riduzione del rischio di ictus ed eventi ischemici e riduzione del rischio di PAD (peripheral artery disease); 
- riduzione del rischio di demenza e miglioramento delle funzioni cognitive rispetto ai non bevitori.

Il problema da porsi è sapere quando e quanto bere, e poter scegliere, nella consapevolezza di tutte le conseguenze derivanti dall'uso e dall'abuso di bevande alcoliche.

2.17 Alcuni studi recenti hanno mostrato che i cambiamenti dei consumi di bevande alcoliche sono quasi sempre dipendenti in misura prevalente da fattori socioeconomici, demografici e culturali e, in misura variabile, - ma minore rispetto agli altri fattori di contesto - dall'introduzione di politiche restrittive, specie quelle che limitano luogo e tempo di accesso ai consumi, l'età legale di acquisto delle bevande alcoliche, il bere alla guida. Più spesso di quanto non ci si attenda, la riduzione dei consumi è comparsa in assenza di politiche di prevenzione ${ }^{12}$.

2.18 In relazione a quanto disponibile in letteratura alcuni studiosi hanno ipotizzato che in Italia possa operare un fenomeno di autoregolazione immaginabile come un insieme di codici sociali e di percorsi di adattamento individuale e collettivo che influiscono sui cambiamenti dei consumi e degli abusi13. Il termine di "autoregolazione" introdotto da De Rita14 significa che una comunità modifica la propria condotta del bere attraverso gli eventi storici, politici, culturali e religiosi che modellano le condotte informali degli individui. Sono necessari ulteriori studi per approfondire questa ipotesi anche in diacronico.

2.19 Per I'Italia quindi ha ancora senso parlare di pratiche di autoregolazione, che possano favorire una riduzione dei consumi e degli abusi di bevande alcoliche. Gli italiani sembrano aver sviluppato le competenze del bere responsabile che si accompagnano a una visione equilibrata del gusto, della qualità della vita, della convivialità e del saper stare insieme agli altri. A questo riguardo, la nota distinzione tra culture asciutte e culture bagnate, storicizzata e attenuata, mantiene una sua indubbia evidenza.

2.20 Riteniamo importante sottolineare ancora una volta la distinzione tra uso e abuso delle bevande alcoliche in una cultura mediterranea. II primo contraddistingue un comportamento associato a regole alimentari e mediato dalla capacità, culturalmente acquisita e socialmente coltivata, di adottare misure di protezione per la propria salute (al limite rinunciando al consumo). II secondo contraddistingue un rapporto patologico continuato con le bevande alcoliche dove viene meno la capacità di controllo, di moderazione e di autolimitazione spesso dovuta a una vulnerabilità di tratto e/o di stato individuale.

2.21 Ne deriva che anche le pratiche di autoregolazione non sono assolute, ma storiche e relative. Come non esiste una sola cultura dell'alcol non esiste nemmeno una sola cultura di autoregolazione. Le autorità pubbliche e sanitarie devono assumere questa complessità nel giudizio degli interventi pertinenti nei diversi contesti e sui diversi destinatari, senza rinunciare a mettere in atto quelle misure necessarie alla correzione di comportamenti che sono a rischio per la collettività e che possono aiutare l'individuo a mantenersi all'interno di un consumo responsabile.

3. L'Osservatorio e i firmatari del presente documento continuano a ritenere valido un modello di controllo dell'eccesso di bevande alcoliche che faccia appello alle risorse razionali, emotive, relazionali e sociali degli individui, e che temperi le scelte e gli esiti sociali dei comportamenti individuali e collettivi.

Sottoscritto da:

Dr. Allaman Allamani - Già Centro Alcologico Azienda Sanita- ria di Firenze

Dr. Giovanni Aresi - Assegnista di ricerca e Professore a contratto, Università Cattolica del Sacro Cuore Milano

Dr. Alberto Arnaudo - Ser.T. Cuneo

Dr. Pierluigi Ascani - Format Research Roma

Dr. Andrea Balbi - Dipartimento di Salute Mentale ASL RMD Roma

Prof. Tito Baldini - Associazione Romana di Psicoterapia dell'Adolescenza e del Giovane Adulto (ARPAd)

Dr.ssa Roberta Balestra - Direttore Dipartimento Dipendenze ASS1 Triestina

Dr.ssa Franca Beccaria - Società Eclectica Torino

Prof. Carlo Buzzi - Dipartimento di Sociologia e Ricerca Sociale, Università di Trento

Prof. Stefano Canali - Scuola Internazionale di Studi Superiori Avanzati (SISSA) Trieste

Dr.ssa Luciana Castelli - Dipartimento di Formazione e Apprendimento della Scuola Universitaria Professionale della Svizzera Italiana Locarno

Prof.ssa Angela Cattaneo - Dipartimento di Comunicazione e Ricerca Sociale, Università La Sapienza Roma

Prof. Fortunato Ciardiello - Presidente ESMO (European Society for Medical Oncology)

Dr. Mauro Cibin - Ser.T. di Dolo USL 13 Mirano (VE)

Prof. Costantino Cipolla - Dipartimento di Sociologia e Diritto dell'Economia, Università di Bologna

Prof. Massimo Clerici - Dipartimento di Medicina e Chirurgia, Università Milano Bicocca

Dr. Alessandro Coacci - Past President FederSerD

Dr. Maurizio Coletti - Società Itaca Italia Roma Dr.ssa Carla Collicelli - Fondazione CENSIS Roma

Dr. Michele Contel - Segretario Generale Osservatorio Permanente sui Giovani e l'Alcol Roma

Dr. Augusto Consoli - Azienda Sanitaria Locale Torino 2

Prof. Amedeo Cottino - Già Professore Sociologia del Diritto, Università di Torino

Dr. Edoardo Cozzolino - Direttore SC SerT 1 Dipartimento delle Dipendenze ATS della Città Metropolitana di Milano

Prof. Giovanni De Gaetano - Dipartimento di Epidemiologia e Prevenzione IRCCS Istituto Neurologico Mediterraneo Neuromed Pozzilli (IS)

Dr. Francesco De Matteis - Coordinatore Distretti Sanitari Azienda Sanitaria Provinciale di Reggio Calabria - Regione Calabria Prof. Fausto D'Egidio - Presidente Nazionale Federserd Prof.ssa Valeria del Balzo - Dipartimento di Medicina Sperimentale, Università La Sapienza Roma

Dr. Marco Faccini - Azienda Ospedaliera Alta Specialità in Alcologia AOUI Verona

Dr. Guido Faillace - Dipartimento Dipendenze Alcamo Trapani Dr. Pino Faro - Direttore SC Ser.T. Sangone ASL TO3

Prof. Maurizio Fea - Federserd Pavia

Prof.ssa Maria Pia Garavaglia - Già Ministro della Salute

Prof. Piernicola Garofalo - Presidente Società Italiana Medicina dell'Adolescenza

Prof. Vincenzo Gerbi - Scienza e Tecnologia degli Alimenti, Università di Torino

Dr.ssa Francesca Guarino - Dipartimento di Sociologia e Diritto dell'Economia, Università di Bologna

Dr.ssa Ina Hinnenthal - Asl 1 Imperia

Prof. Lugi Janiri - Facoltà di Medicina e Chirurgia, Università Cattolica del Sacro Cuore Roma

Prof.ssa Michaela Liuccio - Dipartimento di Comunicazione e Ricerca Sociale, Università La Sapienza Roma 
Prof.ssa Carmelina Loguercio - Facoltà di Medicina e Chirurgia, Seconda Università di Napoli

Prof. Alfio Lucchini - Già Presidente Nazionale Federserd - Milano Dr.ssa Sabrina Molinaro - Istituto di Fisiologia Clinica CNR di Pisa

Dr. Felice Nava - Responsabile Sanità Penitenziaria - Responsabile SSD ULSS 16 Padova

Prof. Franco Prina - Sociologia giuridica e della devianza, Università di Torino

Dr. Andrea Poli - Presidente Nutrition Foundation of Italy Milano Dr. Daniele Rossi - Confagricoltura - Coordinatore Europeo delle Piattaforme Tecnologiche Nazionali Food For Life Roma Dr.ssa Gianna Sacchini - Direttore Servizio Territoriale Dipendenze Patologiche Area Vasta 4 Fermo

Prof. Massimo Santinello - Dipartimento Psicologia dello Sviluppo e Socializzazione, Università di Padova

Prof. Vincenzo Savarino - Dipartimento di Medicina Interna e Specialità Mediche, Università di Genova

Prof. Francesco Scotti - Psichiatra, Perugia

Dr.ssa Valeria Siciliano - Istituto di Fisiologia Clinica CNR Pisa

Prof. Bruno Silvestrini - Società Noopolis Roma

Prof. Francesco Sofi - Dipartimento di Medicina Sperimentale e Clinica, Università Ospedale di Careggi Firenze

Dr.ssa Cristina Stanic - Responsabile Infermieristica Dipartimento Dipendenze ASS1 Triestina

Prof. Enrico Tempesta - Presidente Osservatorio Permanente sui Giovani e I'Alcol Roma

Dr. Paolo Ugolini - Già Direttivo Società Italiana di Sociologia della Salute Bologna

Prof. Fulvio Ursini - Dipartimento di Medicina Molecolare, Università di Padova

Prof. Paolo Usai - Università di Cagliari

Dr. Massimo Valsecchi - Direttore Dipartimento Prevenzione ULSS 20 Verona

Prof. Piergiorgio Zuccaro - Già Dirigente Ricercatore Istituto Superiore di Sanità Roma

\section{Note}

1. Seligman M.E.P ( 1998)., Learned optimism. How to change your mind and your life, Simon and Schuster, New York.

2. Vd. Relazione del Ministro della Salute al Parlamento, sugli interventi realizzati ai sensi della legge 30.03.2001 n. 125 "Legge quadro in materia di alcol e problemi alcol correlati", presentata il 22 marzo 2016. 3. Vd. tra l'altro Osservatorio Permanente sui Giovani e l'Alcol-Istituto di Fisiologia Clinica, CNR, Consumo di Bevande alcoliche dei giovani in Italia dal 2000 ad oggi. Una revisione sistematica, 2015.

http://www.alcol.net/it/primo-piano-home-page/consumo-di-bevandealcoliche-dei-giovani-in-italia-dal-2000-ad-oggi-una-revisione-sistematica

4. Allamani A., Anderson P., Einstein S. (eds.) ( 2014), "Drinking patterns in 12 European countries: unplanned contextual factors and planned alcohol control policy measures". Substance Use and Misuse, 49(12): 1505-1728.

5. Rehm J., Shield Kd., Rehm M.X. et al. (2012), Alcohol consumption, alcohol dependence and attributable burden of disease in Europe:potential gains from effective interventions for alcohol dependence, Centre for Addiction and Mental Health, Toronto, Canada.

6. Vd. CNAPA Committee on National Alcohol Policy and Action Plan on Youth Drinking and on Heavy Episodic Drinking (Binge Drinking). 2014-2016 Sept. 2014.
7. Il campo di ricerca è ovviamente assai vasto; l'evoluzione delle tecniche sperimentali, rapida. Una proposta succinta di riscontro bibliografico di primo approccio può includere: Dennis E.L., Thompson P.M. (2013), "Mapping connectivity in the developing brain", Int J Dev Neurosci, 31(7): 524-542 - Baker S.T., Lubman D.I., Yücel M. et al. (2015), "Developmental changes in brain network hub connectivity in late adolescence", J Neurosci, 35 (24): 9078-9087 - Guerri C., Pascual M. (2010), "Mechanisms involved in neurotoxic, cognitive and neurobehavioural effects of alcohol consumption during adolescence", Alcohol, 44(1): 15-26 - Paus T. (2005), "Mapping brain maturation and cognitive development during adolescence", Trends Cogn Sci, 9(2): 60-68.

8. Su questi aspetti è interessante: Bessant J. (2008), "Hard wired for risk: neurological science, the 'adolescent brain' and developmental theory", J Youth Stud, 12(3): 347-360.

9. LARN - Livelli di Assunzione di Riferimento di Nutrimenti ed energia per la popolazione italiana. IV revisione. A cura della Società Italiana di Nutrizione Umana (SINU), 2014.

10. Andréasson S., Allebeck P. (2005), "Alcohol as medication is no good. More risks than benefits according to a survey of current knowledge", Lakartidningen, 102(9): 632-637.

11. Di Castelnuovo A., Costanzo S., Bagnardi V. et al. (2006), "Alcohol dosing and total mortality in men and women: an updated meta-analysis of 34 prospective studies", Arch Intern Med, 166(22): 2437-2445 Wakabayashi I. (2016), "A U-shaped relationship between alcohol consumption and cardiometabolic index in middle-aged men", Lipids Health Dis, 15(1): 50 - Dal Maso L., Torelli N., Biancotto E. et al. (2015), "Combined effect of tobacco smoking and alcohol drinking in the risk of head and neck cancers: a re-analysis of case-control studies using bidimensional spline models", Eur J Epidemiol, 31(4): 385-393, 2015 Giacosa A., Barale R., Bavaresco L. et al. (2016), "Mediterranean way of drinking and longevity", Crit Rev Food Sci Nutr, 56(4): 635-640 - Poli A., Marangoni F., Avogaro A. et al. (2013), "Moderate alcohol use and health: a consensus document", Nutr Metab Cardiovasc Dis, 23(6): 487504 - Chiva-Blanch G., Arranz S., Lamuela-Raventos R.M. et al. (2013), "Effects of wine, alcohol and polyphenols on cardiovascular disease risk factors: evidences from human studies", Alcohol Alcohol, 48(3): 270277 - Abramson J.L., Williams S.A., Krumholz H.M. et al. (2001), "Moderate alcohol consumption and risk of heart failure among older persons", JAMA, 285(15): 1971-1977 - Chen J.Y., Zhu H.C., Guo Q. et al. (2016), "Dose-dependent associations between wine drinking and breast cancer risk - Meta-analysis findings", Asian Pac J Cancer Prev., 17(3): 1221-1233 - Wang Y.T., Gou Y.W., Jin W.W. et al. (2016), "Association between alcohol intake and the risk of pancreatic cancer: a doseresponse meta-analysis of cohort studies", BMC Cancer, 16(1): 212.

12. Bernstein K.T., Galea S., Ahern J. et al. (2007), "The built environment and alcohol consumption in urban neighborhoods", Drug Alcohol Depend, 91(2-3): 244-252 - Ward B.W. (2011), "Identifying environmental effects on alcohol use and social norms. The socio-environmental context model", J Hum Behav Soc Environ, 21(5): 505-520 - Nelson J.P., McNall A. (2015), "What happens to drinking and harms when alcohol policy changes? A systematic review of five natural experiments for alcohol taxes, prices and availability", Social Science Research Network, June 1 - Allamani A., Pepe B., Baccini M, et al. (2014), "Europe. An analysis of changes in the consumption of alcoholic beverages: the interaction between consumption, related harms, contextual factors and alcoholic beverage control policies", Subst Use Misuse, 49(12): 1692-1715.

13. Cipriani F., Prina F. (2006), "I risultati della ricerca. Sintesi finale", in Allamani A., Cipriani F., Prina F. (eds), I cambiamenti nei consumi di bevande alcoliche in Italia. Uno studio esplorativo sul decremento dei consumi negli anni 1970-2000, Osservatorio Permanente sui Giovani e L'Alcol, Quaderno 17, Litos, Roma: 275-290.

14. Vd. Prefazione di Giuseppe de Rita a: Rossi D. (1992), Alcol: Consumi e politiche in Europa, Otet, Roma. 